\title{
Introduction of Major Institutions
}

\author{
A Brief Introduction to \\ Research Center for the Civilization \\ of Chinese Characters of Zhengzhou University (ZZU)
}

\section{Yunfu*}

As the cradle of Chinese civilization and the hometown of Chinese characters, Henan province not only has rich Chinese character resources, but also has a long tradition of Chinese character research. In order to preserve the culture of the Central Plains and carry forward the civilization of Chinese characters, Zhengzhou University has introduced an innovative team led by Changjiang Scholar professor Li Yun-fu. On September 26, 2016, the Research Center for the Civilization of Chinese Characters of ZZU was formally established, with Professor Li Yun-fu as the director. Since its establishment, the center has made remarkable achievements in team building, platform construction, international exchange, scientific research achievements, hosting conferences and academic lectures, talent training and many other aspects.

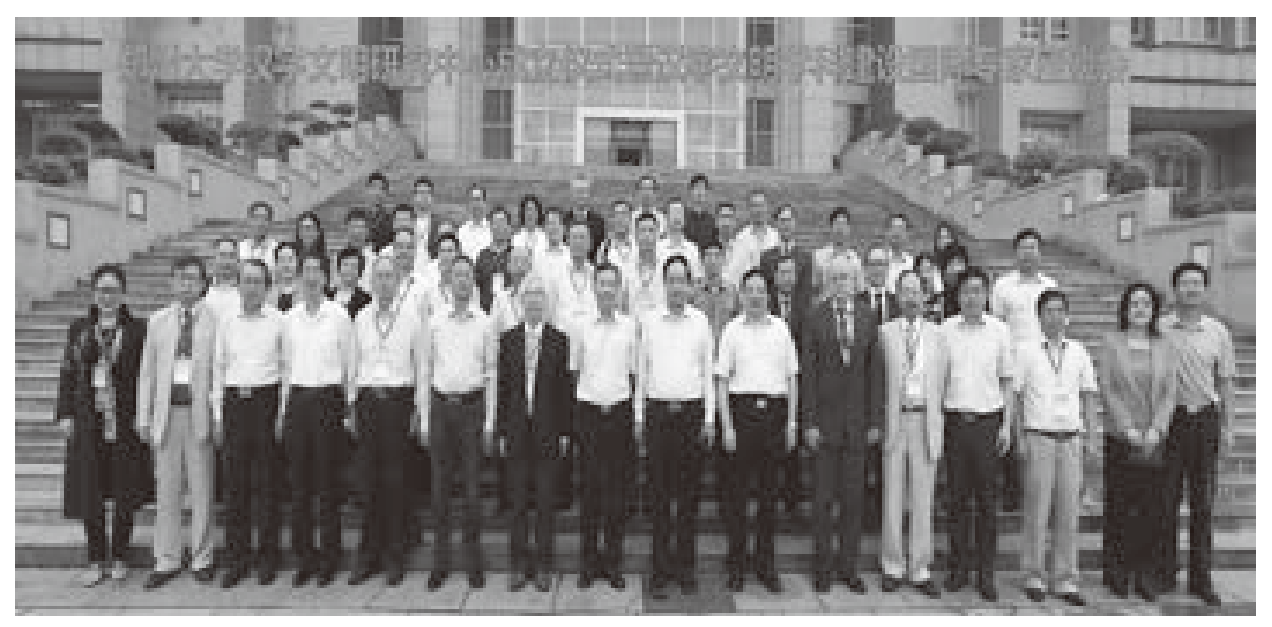

Photo taken at the founding ceremony

* LI Yunfu 李運富 is Professor and Director of Research Center for the Civilization of Chinese Characters of Zhengzhou University 


\section{Research Area}

Research Center for the Civilization of Chinese Characters of ZZU has four main research directions.

\section{(1) Theory and History of Chinese Characters}

The research contents are as follows: The Three-Planes Theory of Chinese Graphology, among which function of Chinese characters is the main emphasis of our research; the evolution history of Chinese characters font, function of Chinese characters, and of Chinese characters recording specific words and phrases.

\section{(2) Ancient Writing and Traditional Culture}

The research contents are as follows: Ancient writing materials including oracle bone and bronze inscriptions, bamboo slips and stele inscriptions, as well as the historical and cultural elements involved in the above materials.

\section{(3) The spread of Chinese Characters and Overseas Chinese Characters}

The research contents are as follows: The spread, variation phenomenon and current situation of Chinese characters in ethnic minority areas and all around the world, , quasi Chinese characters, as well as the comparison between Chinese characters and non-Chinese characters.

\section{(4) Shuo Wen Jie Zi and Education of Chinese Characters}

The research contents are as follows: Shuo Wen Jie Zi and related research, study of six categories of Chinese characters, education of Chinese characters in primary and secondary schools, international Chinese characters education, etc.

\section{Theoretical Features}

Research Center for the Civilization of Chinese Characters of ZZU is characterized by "The Three-Planes Theory of Chinese Characters", advocating that Chinese characters have three properties of their own: shape, constitution and function. Accordingly, three branches of Chinese characters research should be established, that is, the study of Chinese graphic shape, the study of Chinese graphic constitution and the study of Chinese graphic function, thus, it forms a new system of three-dimensional Chinese characters study. The study of Chinese graphic function is one of the three planes of Chinese characters, but it has been neglected for a long time. In recent years, the team led by professor Li Yun-fu has done a lot of work in this field, with relevant achievements including "New Perspective on Theory of Chinese Characters" (Beijing Normal University Press, 2012), "Research on Function 
of Chinese Characters, Theory and Application" and "Research on Function of Chinese Characters, Investigation on the Phenomenon" (China Social Science Press, 2016).

At the same time, Research Center for the Civilization of Chinese Characters of ZZU actively advocates the "Cross-Cultural Chinese Character Research", and emphasizes that the study of Chinese characters should have a cross-cultural perspective in addition to its own cultural perspective. The "Cross-Cultural Chinese Character Research" has two meanings: one is treating cross-culture as a method and perspective of research, in other words, studying Chinese characters from a cross-cultural perspective; the other is treating "cross-cultural Chinese character" as an object of the research, that is to say, studying Chinese characters in different cultural contexts. Relevant achievements include "A Study on Cross-Cultural Chinese Characters" (Journal of Beijing Normal University (Social Sciences), 2018(1)), "CrossCultural Vision and Chinese Character Research" ( Social Sciences Academic Press, 2018), etc.

\section{Research Team}

Research Center for the Civilization of Chinese Characters of ZZU now has 19 full-time researchers, including Professor Li Yun-fu, Huang Xi-quan, Zhang Su-feng, He Hua-zhen, Wu Guo-sheng, Qi Hang-fu, Zhang Qing-song, Yu Shao-hong and Wang Jing (including 6 specially invited professors of Zhengzhou University); associate professors Wen Min, Liu Feng-hua, He Qing, Miao Li-juan, Liu Qiu-rui, Zhang Xin-jun, etc; besides, the center employed Hong De-rong, He Yu-hua, Wang Kai-bo, Niu Zhen and other lecturers. In September 2016, the first academic committee was established, Research Center for the Civilization of Chinese Characters of ZZU hired a number of visiting researchers from abroad, Hong Kong, Macao and Taiwan, as well as plenty of part-time researchers from Chinese mainland.

Research Center for the Civilization of Chinese Characters of ZZU participates actively in the development of Historical and Cultural Heritage in Central Plain against the background of "Double First-rate" construction. The application of first-level doctoral degree in "Chinese Language and Literature" was approved successfully, and was confirmed as key discipline of Henan first-level discipline. The "Theory and History of Chinese Characters" team was selected as the philosophy and social science innovation team in Henan institutions of higher learning. The "Chinese Character Inheritance and Education Research Center" jointly built by Research Center for Civilization of Chinese Characters of ZZU, the National Language Commission and the Education Department of Henan Province was passed by expert evaluation and will be put into construction soon. 


\section{Academic Achievements}

In order to timely launch the research results obtained by the members of the center, the center has successively edited and published the "Chinese character civilization research" series. The series include the conference anthologies organized by the center or center members (including part-time workers). Up till today, several anthologies have been published, such as Studies on Han Books in East Asia and Han Nan Ancient Dictionaries in Vietnam edited by He Hua-zhen and Ruan Jun-qiang, and Studies on CrossCultural Perspectives and Chinese Characters edited by Li Yun-fu. The series of books on the study of Chinese character civilization includes monographs, co-authors, data collation, reference books, thematic series and teaching materials by members of the center (including part-time workers). For example, Notes on the Chu Bamboo Slips in the Shanghai Museum edited by Yu Shao-hong, He Hua-zhen's Research on the Spread of Popular Form of Characters Abroad, Liang Xiao-hong's Research on Japanese Characters -the Sound and Meaning of Japanese Buddhist Sutras, etc. In addition, members of the center have published many papers and other works in academic journals at home and abroad. Such as Li Yun-fu's Research on Function of Chinese Character, Theory and Application and Research on Function of Chinese Characters, Investigation on the Phenomenon (China Social Science Press, 2016), the Korea version of Three-Dimensional Research on Chinese Characters, the Vietnamese edition of New Perspective on Theory of Chinese Characters and Comments on Chinese Characters Sequel; Zhang Su-feng's Interesting Pictorial Book of Chinese Characters and Viewing China by Chinese Characters; Wen Min's Annotation of Chen Zhou Zhi of Shun Zhi Version; Zhang Qing-song's Research on the Variant Chinese Characters in Zheng Zi Tong and Plain Words and Pictorial Explanations of Kao Gong Ji; Miao Li-juan's Chinese Zodiac Animals in Chinese Characters, etc.

\section{Scientific Research Projects}

The Research Center for the Civilization of Chinese Character undertakes many scientific research projects at the national level, provincial and ministerial-level, including 20 original projects (obtained before the establishment of the center) and 15 new projects (obtained after the establishment of the center). Such as National Social Science Fund Major Project "Database Construction and Research on Ancient and Modern Characters" and major project entrusted by the Ministry of Education and the National Language Commission "Review the 120 Years of Oracle-bone Inscriptions Discovery (Memorial Atlas and Research Overview)" presided over by professor Li Yun-fu, National Social Science Fund Major Project "Vietnamese Chinese 
Character Resources and Related Research" presided over by professor $\mathrm{He}$ Hua-zhen, National Social Science Fund Major Project "A Dictionary for the Study of Oracle-bone Inscriptions" presided over by professor Huang Xi-quan and so on. All of these research projects will make a series of research achievements.

\section{Academic Conferences and Lectures}

Since its establishment, the Research Center for the Civilization of Chinese Character of ZZU has hosted 8 international and domestic academic conferences: International Expert Symposium on the Construction of Chinese Character Civilization (2016.9), Symposium on the Achievements of National Social Science Fund Major Project "Compilation of Ancient and Modern Characters in Annotations of all Ages "(2017.2), Expert Symposium on Museum of the Spread of Chinese Characters (2017.2), International Symposium on Han Books in East Asia and Han Nan Ancient Dictionaries in Vietnam (2017.5), Symposium on the History and Current Situation of Using Chinese Characters in Ethnic Areas (2018.3), Opening Discussion Meeting of National Social Science Fund Major Project "Vietnamese Chinese Character Resources and Related Research" (2018.5), Symposium on Theory and History of Chinese Characters (2018.5), and the First International Symposium on Cross-cultural Chinese Characters (2018.10). In addition, the 10th Annual Conference of the Chinese Character Association will be undertaken by our center in October of the following year.

Since its establishment, the Research Center for the Civilization of Chinese Character of ZZU has holded "Cross-cultural research high-end forum" series of academic lectures and has invited 20 experts from China and abroad to give lectures.

\section{Academic Journal}

The Journal of the Study of Chinese Characters and Language can be traced back to its predecessor Journal of Language Knowledge, which was first published in 1985 and was changed to the current name by the State Administration of Press, Publication, Radio, Film and Television (SAPPRFT) in 2017. The journal is supervised and hosted by Zhengzhou University, undertaken by the Civilization of Chinese Character Seminars and College of Liberal Arts of Zhengzhou University and co-organized by the Chinese Character Association, the Chinese Ancient Character Research Association as well as the Historical Linguistics Research Branch of Chinese Linguistic Association. The International standard serial numbering for this journal is: ISSN 2096-4986 and Domestic unified serial numbering for it is: CN41-1450 $/ \mathrm{H}$. Now, the chief editor of this journal is professor Li Yun-fu, the chairman 
of the academic committee is professor Huang De-kuan and the director of the editorial committee is professor Zhang Yong-quan. This journal mainly publishes the research results of Chinese characters and unearthed documents, as well as the research achievements of Chinese language history and modern Chinese, including but is not limited to the contents of theory and history of Chinese characters, ancient writing and traditional culture, Shuowen and Liushu (six categories of Chinese characters), the spread of Chinese characters and overseas Chinese characters, comparison of Chinese characters, relationships between Chinese characters and words, interpretation of difficult words, standardization and teaching of Chinese character, evolution of Chinese language, etc.

Editorial Office Correspondence Address: 450001 Editorial Office of The Study of Chinese Characters and Language, Research Center for the Civilization of Chinese Character, Zhengzhou University, No. 100 Science Avenue, Zhengzhou City, Henan Province, People's Republic of China (PRC).

E-mail for Submission: hzwm2018@126.com.

\section{Digital museum of the spread of Chinese Characters}

The spread of Chinese characters has a long history and involves a wide range of fields, including a rich variety of documents and cultural relics. But there still have no fixed platform to store and display them. Therefore, the Research Center for the Civilization of Chinese Character plans to lead the establishment of "Digital Museum of the Spread of Chinese Characters", aiming to make a comprehensive collection of various Chinese characters and cultural relics transmitted and produced during the spreading of Chinese characters. At the same time, we will use collection, photography, video, animation, rubbing as well as other methods to collect and display these Chinese character resources with the nature of spreading, making this museum to be the most comprehensive and most convenient world-wide digital Chinese character resources database.

\section{Cooperation and Exchanges}

The Research Center for the Civilization of Chinese Character of ZZU attaches great importance to academic cooperation between fraternal institutions, being committed to the construction of "three circles of friends", namely the circle of Mainland friends, the circle of Hong Kong, Macao and Taiwan friends, and the circle of international friends. In the circles, cooperation will be carried out on resources sharing, activities co-organized, research mutual assistance, achievements mutual promotion as well as visits and 
personnel exchanges. Many fraternal institutions, until now, have established cooperative relationships with our center or have intention of cooperation, for instance, Research Center of Overseas Chinese Character Culture, Shanghai Jiao Tong University; Wu Yu-zhang Language and Character Research Center, Renmin University of China; Research Center of Overseas Chinese Characters, Zhejiang University of Finance \& Economics; Institute of Chinese Language and Literature, Southwest University; Institute of Chinese Characters in Korea, Kyungsung University; Institute of Han Nan Literature, Vietnam Academy of Social Sciences; Research Center of Chinese Character Resources in East Asia; College of East Asia, Ruhr-University Bochum; Research Institute of Literature, Hokkaido University; Research Institute of East-West Academic, Kansai University; Institute of Chinese Character Culture, Fukuoka International University; National Museum of Chinese Writing (NMCW); Luoyang Museum of Metal and Stone Writing; Luoyang Museum of Stone Writing of Nine Dynasties, etc. Now, the center is launching the establishment of "the League of Nations for Cross-cultural Chinese Character Research", and more than 10 foreign and domestic institutions have agreed to join. We also sincerely hope that more teams and institutions will join us.

In addition, the center has Co-established the Research Base for Ethnic Cross-Cultural Chinese Characters in Southwest region with the Xingyi Normal University for Nationalities (Guizhou), Research Base for Inheritance and Spread of Chinese Character Civilization of ZZU with National Museum of Chinese Writing (Anyang), and Research Base for the Civilization of Chinese Character of ZZU with Research Center of Xu Shen Culture (Luohe), Luoyang Museum of Metal and Stone Writing (Luoyang) and Henan Museum(Zhengzhou), etc.

Web Site: http://www5.zzu.edu.cn/hzwm/

Contact Number: 0371-67783163

E-mail Address: zdhzwmzx@126.com

Correspondence Address: 450001 Research Center for the Civilization of Chinese Character, Zhengzhou University, No. 100 Science Avenue, Zhengzhou City, Henan Province, People's Republic of China. 


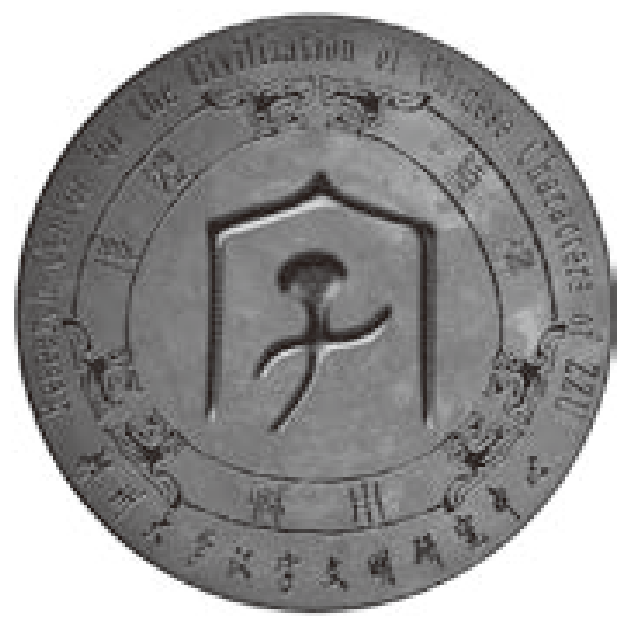

Research center logo

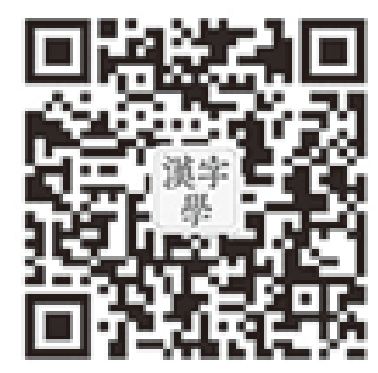

Official WeChat QR code

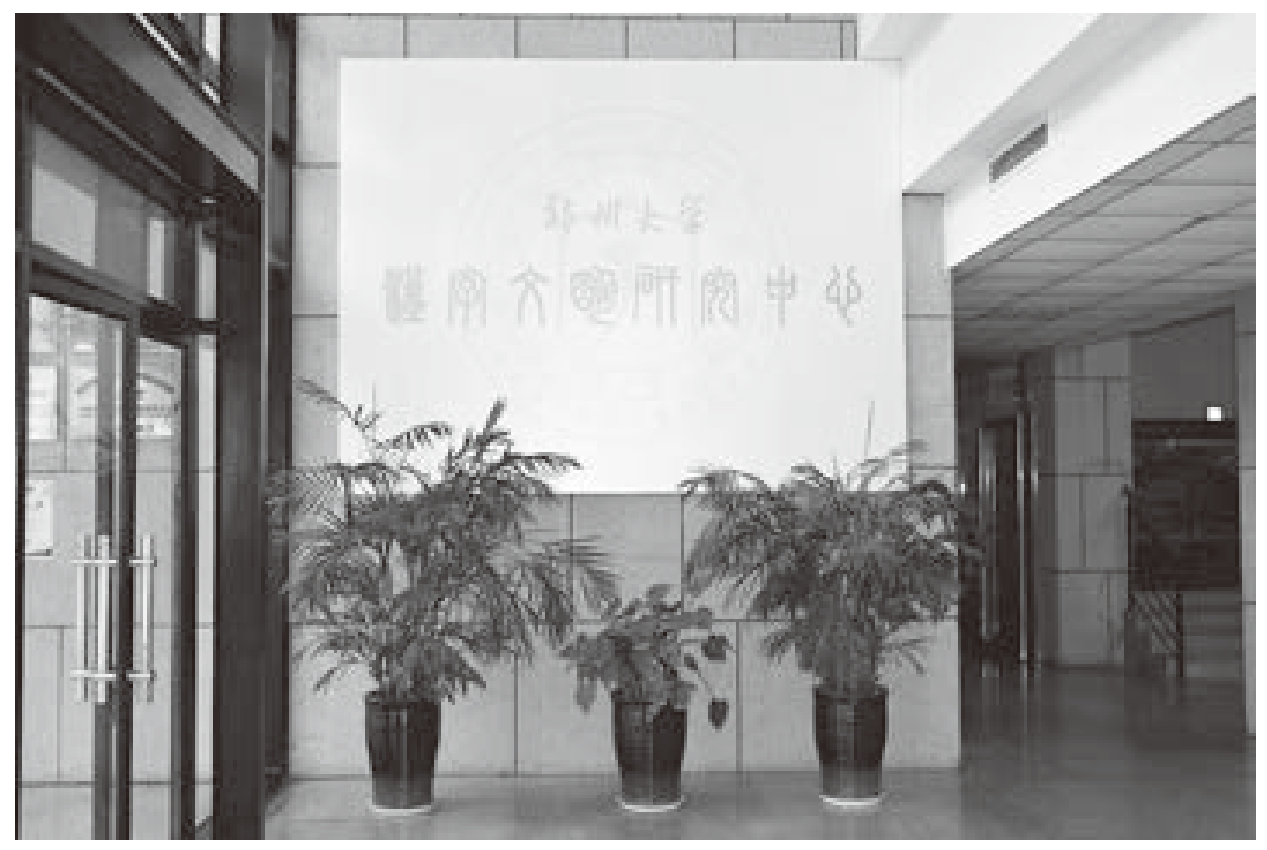

The outdoor scene 
A Brief Introduction to Research Center for the Civilization of Chinese Characters of Zhengzhou University (ZZU) 99

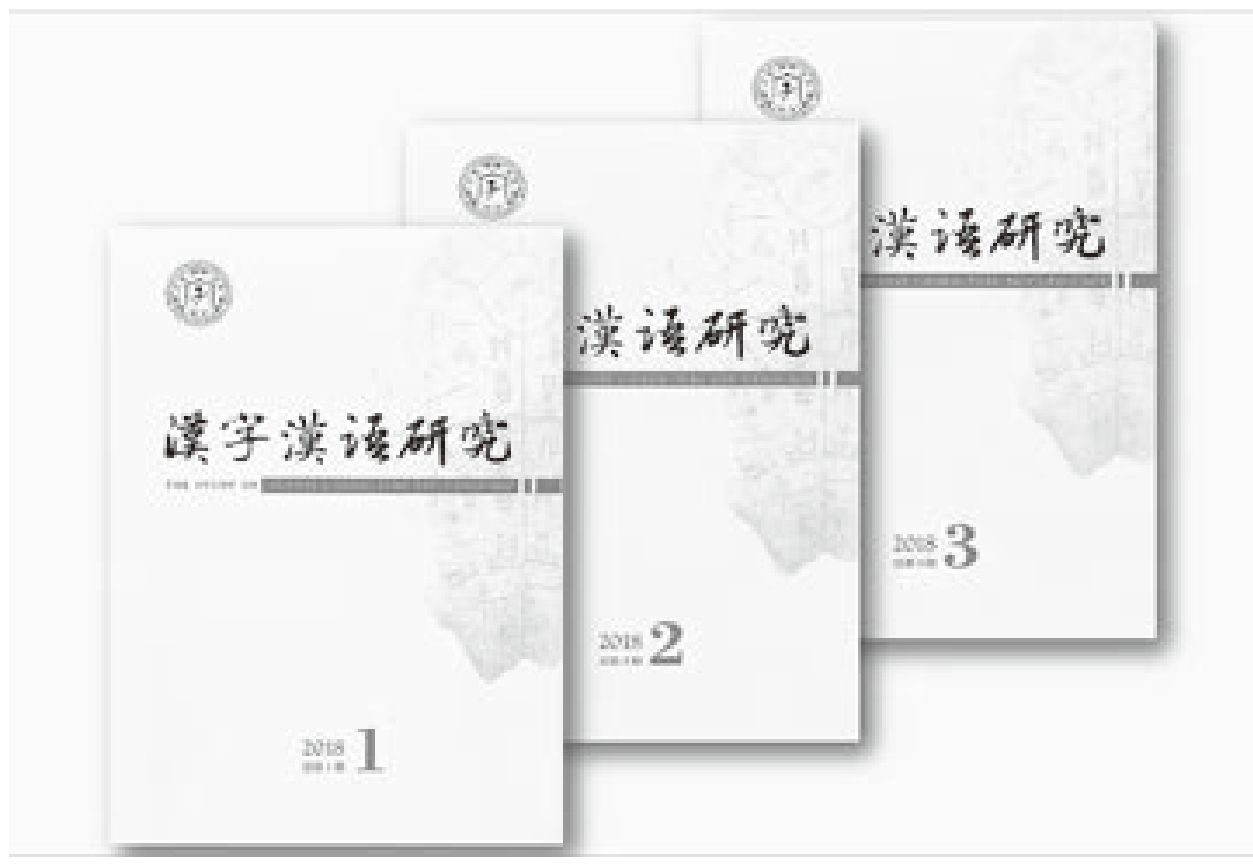

The Journal of the Study of Chinese Characters and Language

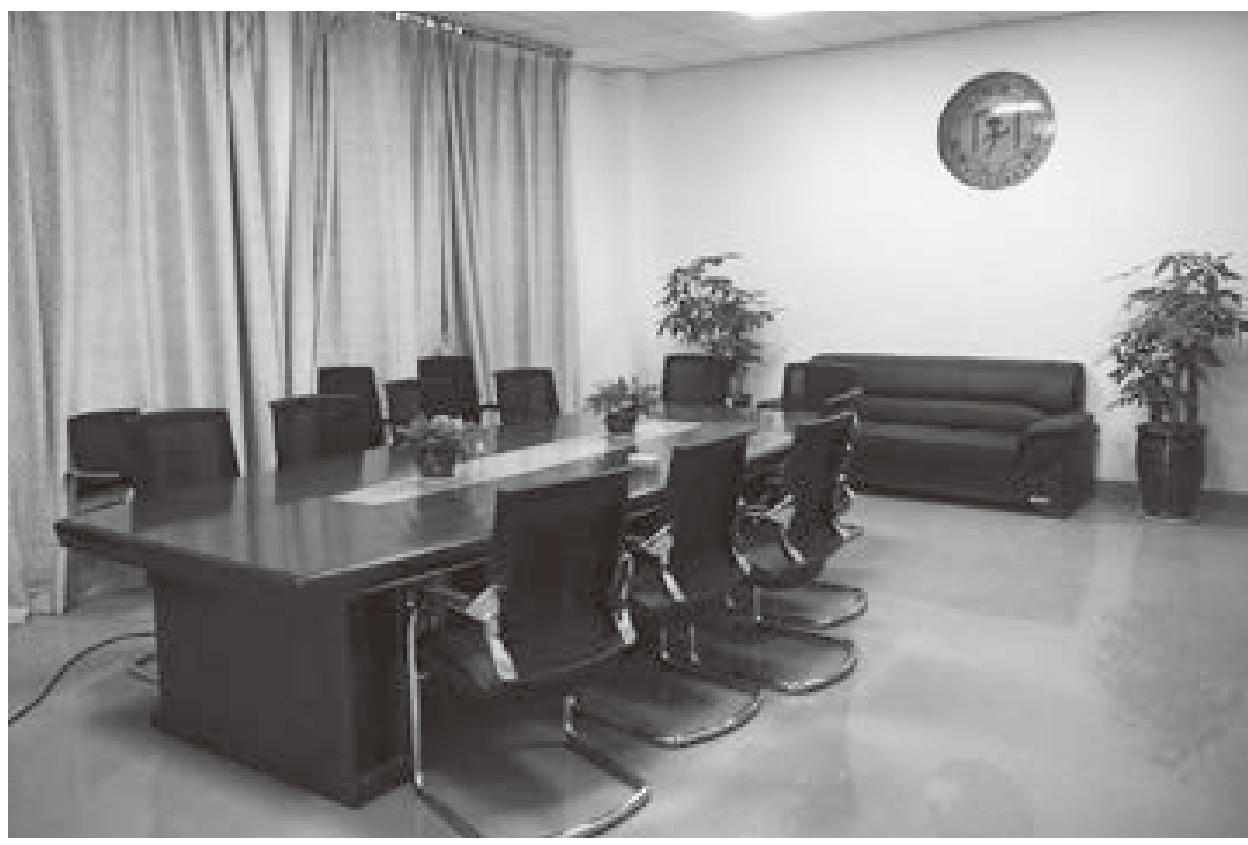

The conference room 


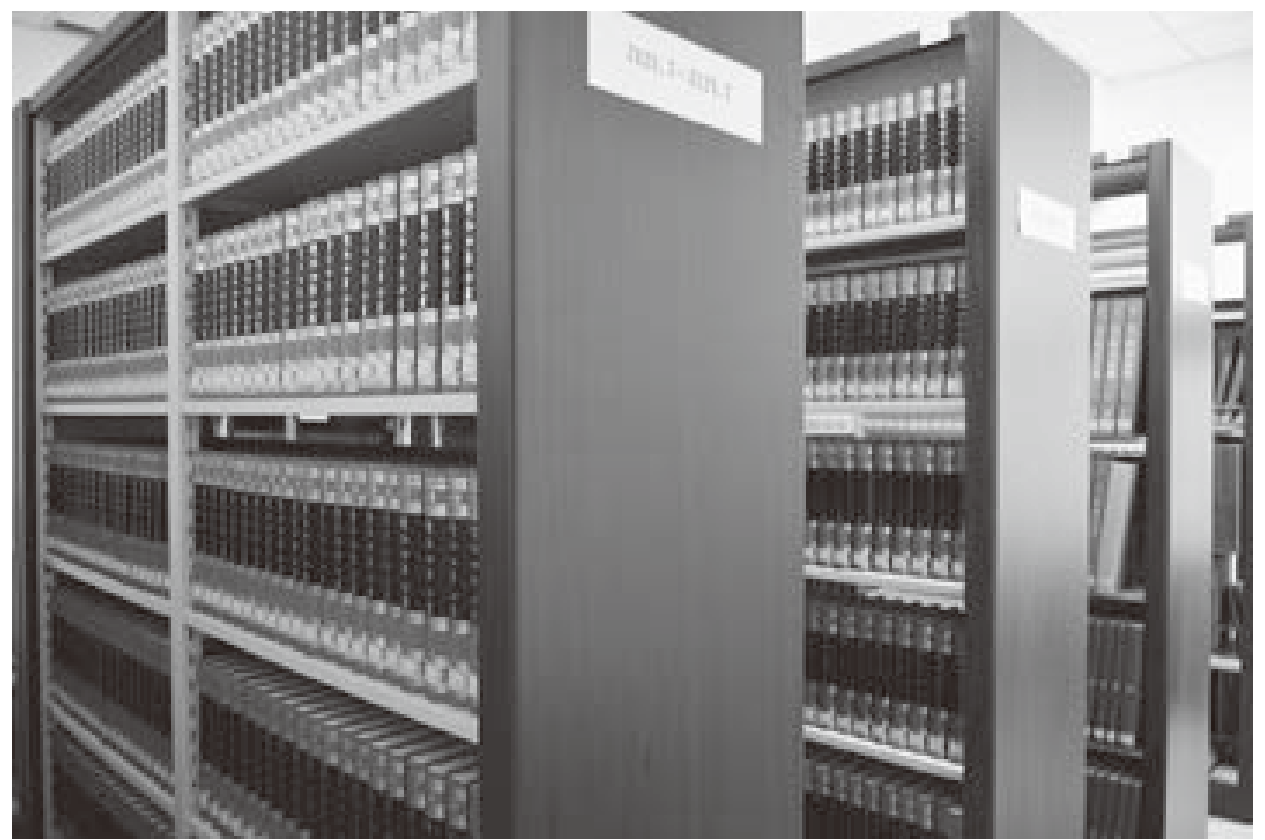

The professional library

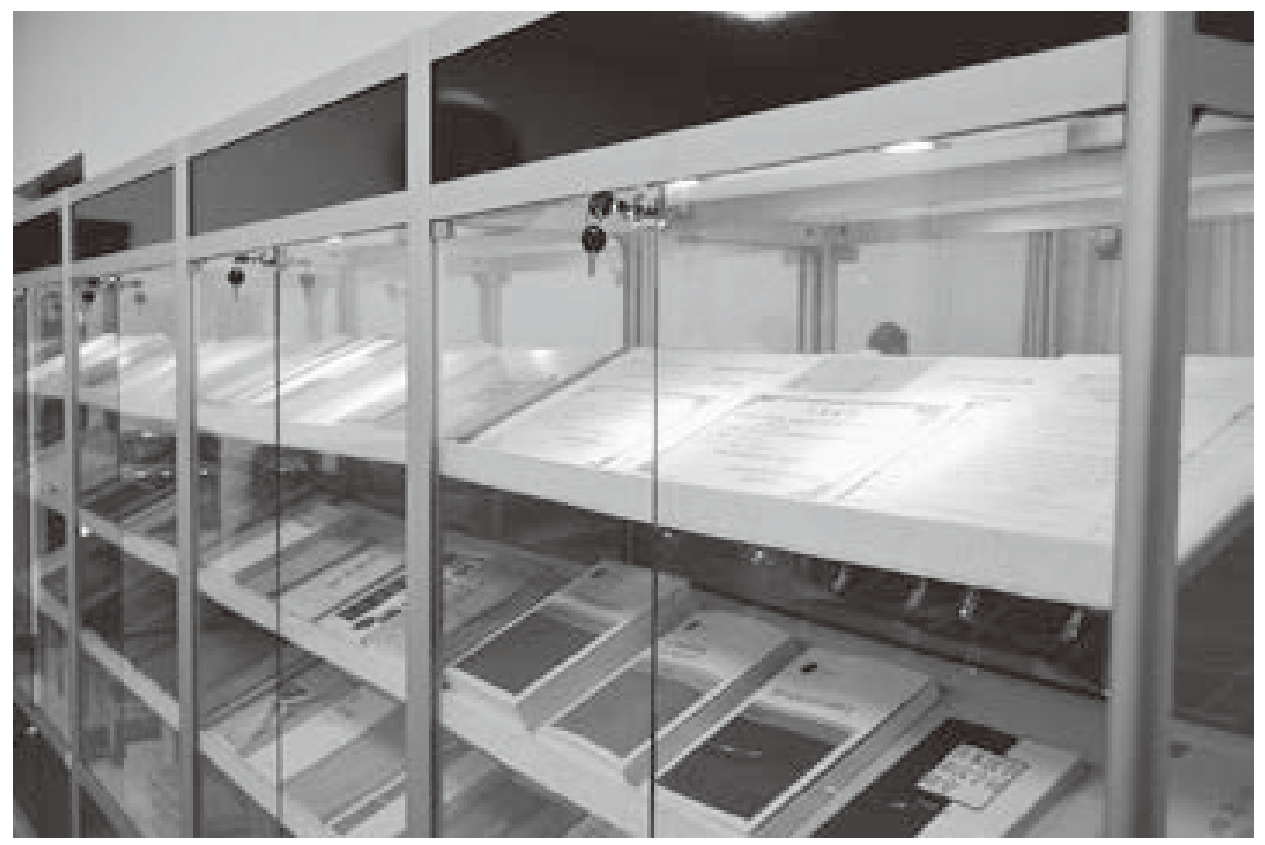

Academic achievements 